\title{
Evaluation of Clinical Laboratory Tests' Turnaround Time in a Tertiary Hospital in Democratic Republic of the Congo
}

\author{
Chabo Byaene Alain ${ }^{*}{ }^{(}$, Mabela Makengo Matendo Rostin ${ }^{2}$, Konde Nkiama Numbi Joël3, \\ Muhindo Mavoko Hypolite ${ }^{4}$, Kayembe Nzongola-Nkasu Donatien', Tanon Aristophane Koffi ${ }^{5}$, \\ Muwonga Masidi Jérémie ${ }^{1}$, Situakibanza Nani-Tuma Hippolytee ${ }^{4,6}$
}

\author{
${ }^{1}$ Department of Medical Biology, University Hospital of Kinshasa, Faculty of Medicine, University of Kinshasa, Kinshasa, \\ Democratic Republic of the Congo (DRC) \\ ${ }^{2}$ Department of Mathematics and Informatics, Faculty of Sciences, University of Kinshasa, Kinshasa, \\ Democratic Republic of the Congo (DRC) \\ ${ }^{3}$ Department of Health and Environment, Faculty of Medicine, University of Kinshasa, Kinshasa, \\ Democratic Republic of the Congo (DRC) \\ ${ }^{4}$ Department of Tropical Medicine, University Hospital of Kinshasa, Faculty of Medicine, University of Kinshasa, Kinshasa, \\ Democratic Republic of the Congo (DRC) \\ ${ }^{5}$ Department of Dermatology and Infectiology, Faculty of Medical Sciences, Félix Houphouët Boigny University, Abidjan-Cocody, \\ Côte d'Ivoire \\ ${ }^{6}$ Department of Internal Medicine, University Hospital of Kinshasa, Faculty of Medicine, University of Kinshasa, Kinshasa, \\ Democratic Republic of the Congo (DRC) \\ Email: ^byaene_2005@hotmail.com
}

How to cite this paper: Alain, C.B., Rostin, M.M.M., Joël, K.N.N., Hypolite, M.M., Donatien, K.N.-N., Koffi, T.A., Jérémie, M.M. and Hippolyte, S.N.-T. (2021) valuation of Clinical Laboratory Tests' Turnaround Time in a Tertiary Hospital in Democratic Republic of the Congo. Journal of Biosciences and Medicines, 9, 96-111. https://doi.org/10.4236/jbm.2021.97011

Received: June 15, 2021

Accepted: July 12, 2021

Published: July 15, 2021

Copyright $\odot 2021$ by author(s) and Scientific Research Publishing Inc. This work is licensed under the Creative Commons Attribution International License (CC BY 4.0).

http://creativecommons.org/licenses/by/4.0/

c) (i) Open Access

\begin{abstract}
The delay in the delivery of laboratory results can be fatal and can even lead to the death of patients. This study was conducted at the clinical laboratory of the University Hospital of Kinshasa (UHK) from October 2020 to April 2021, aimed to evaluate the laboratory tests' turnaround time (TAT) and to identify reasons for delay. TAT was quantified using a time and motion analysis approach. The evaluation of TAT consisted of comparing the overall intra-lab TAT with the suggested TAT using student t-test at $95 \%$ confidence intervals. Brainstorming was the root cause analysis tool used for identifying reasons for delay. In this study, the laboratory tests' TATs were significantly higher ( $\mathrm{p}$ $<0.001$ ) comparing to international guidelines (60 minutes) and customers' suggested TAT (120 minutes). Only $0.98 \%$ of the samples were reported within 60 minutes of patient reception and $1.47 \%$ within 120 minutes, i.e. an outlier rate of $98.5 \%$. Root causes of delay related to Machinery, Management, Manpower, Materials, Method and Milieu. Because of many reasons, the laboratory is not meeting the established TAT. Preventive and curative measures must be undertaken to reduce the delay and improve the TAT.
\end{abstract}




\section{Keywords}

Time-Motion Analysis, Turnaround Time, Clinical Laboratory, Quality Assurance, Value Stream Mapping, Democratic Republic of the Congo

\section{Introduction}

The healthcare industry (also called the medical industry or health economy) is an aggregation and integration of sectors within the economic system that provides goods and services to treat patients with curative, preventive, rehabilitative, and palliative care. Clinical laboratory is essential in all areas of healthcare industry. In today's world, the clinical laboratory is the heart of any hospital or healthcare system. It is responsible for greater than $70 \%$ of medical diagnoses and decisions made by physicians each year. From early detection and diagnosis of disease to individualized treatment plans based on a person's unique genetic makeup, clinical laboratory testing is the key to improving healthcare quality and containing long-term health costs. A physician or other clinician orders lab tests to diagnose, treat, manage, or monitor a patient's condition. The delay in the delivery of laboratory results can be fatal and can even lead to the death of patients, particularly in intensive care or in the emergency department. It is therefore critical that laboratory results are delivered in the shortest amount of time possible to allow rapid diagnosis and management of patients. More timely results in hospital labs have positive implications in at least five key areas: Optimized patient outcomes, reduced costs, better clinician satisfaction, higher patient satisfaction, better competitive position against other labs [1].

Turnaround time (TAT) of laboratory tests is a useful marker of laboratory efficiency and it is used by many clinicians to evaluate the quality of laboratory service [2]. A study revealed that decreasing TAT diminishes emergency department patient length of stay and increases physicians' satisfaction [3]. Therefore, the World Health Organization (WHO) and the International Organization for Standardization (ISO) suggest that clinical laboratory, in consultation with the users, must determine the maximum allowed TAT for each examination performed in the laboratory. The laboratory shall periodically evaluate whether or not it is meeting the established TAT [4]. This is quite difficult because attending doctors consider TAT from the time the test is ordered to results reporting, whereas laboratory personnel usually use specimen receipt to reporting of results as the TAT [5]. However, when the attending physicians were asked about what ideal waiting time was expected for the most commonly ordered tests, $96 \%$ of 330 respondents considered the ideal turnaround time for the laboratory results should be less than 120 minutes, while $4 \%$ consider the ideal to be 180 minutes [6]. In line with the international guidelines, Bilwani., et al. reported 60 minutes as the recommended threshold [7]. Joan Howanitz and Peter Howanitz suggest 60 minutes or less from sample registration to reporting 
for all common laboratory tests under optimal conditions [8]. The aim of this study is to evaluate whether or not the laboratory is meeting the established TAT and to identify reasons for delay. Taking into account the major source of customers' complaints which is unsatisfactory TAT, we hypothesize that the measured laboratory TAT is greater than the expected or suggested TAT. This alternative hypothesis will be confirmed in case of rejection of the null hypothesis which states that the measured laboratory TAT is equal to the expected or suggested TAT.

\section{Materials and Methods}

\subsection{Setting and Study Design}

It was a hospital based prospective study conducted at the clinical laboratory of the University Hospital of Kinshasa (UHK) from October 2020 to April 2021. The UHK is a 565-bed tertiary care teaching hospital. The Department of Laboratory Medicine of the UHK runs two clinical laboratories. One is the laboratory of Microbiology. The other, called laboratory of Clinical biology and which is concerned by the present study, is the main central laboratory with 5 divisions: phlebotomy and sampling, cytohematology and hemostasis, clinical immunology, clinical biochemistry, immunohematology and transfusion. The laboratory of clinical biology is located within the UHK building, and inpatient and outpatient samples are registered and processed in an accessioning area. Samples are then delivered to the testing laboratory and the site of each division, on the same floor.

\subsection{Sampling and Inclusion Criteria}

The sampling was of convenience. A total of 205 samples for each type of analysis chosen were included, resulting in a total of 1025 samples. All specimens submitted to the clinical laboratory with an order for one of the five most commonly ordered tests, namely full hemogram (hematology test), glucose, urea, creatinine and electrolytes (biochemistry tests) between October 2020 and April 2021 were considered for inclusion in this study. Only those samples that were received by the laboratory from $08: 00 \mathrm{AM}$ to $04: 00 \mathrm{PM}$ were included in the study.

\subsection{Quantifying Turnaround Time}

In this study, TAT is defined as the time taken from patient reception to results availability. This is the intra-laboratory TAT. In fact, the laboratory workflow process consists of twelve steps outlined below (Figure 1).

The laboratory process was divided into three phases. The preanalytic phase included steps 1 (Laboratory tests' ordering), 2 (Laboratory tests' billing and payment), 3 (Patient reception), 4 (sampling = phlebotomy), 5 (sample assemblage), 6 (transit to laboratory), 7 (reception and sorting) and 8 (sample preparation). The analytic phase was step 9 (Manual or automatically sample analysis). The postanalytic 


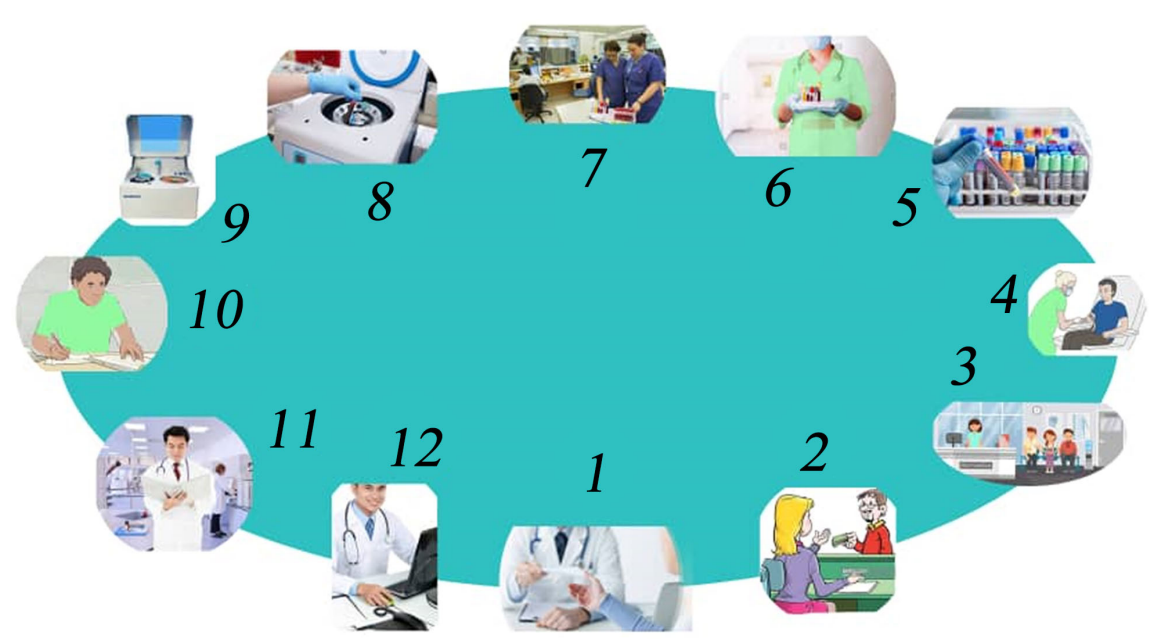

Figure 1. Current Value Stream Mapping (VSM) defining the process of laboratory analysis and identifying all of its components. This is a snapshot capturing how the process is currently being done.

phase included steps 10 (Result reporting), 11 (Biological validation) and 12 (Making the results available). Steps 1 and 2 are extra-laboratory. Intra-lab TAT begins by step 3 .

First step: Laboratory tests' ordering. Once the attending physician has completed the physical examination, he gives the laboratory test request to the patient.

Second step: Laboratory tests' billing and payment. The patient makes lab tests payment at the bank counter.

Third step: Patient reception. After test billing, patients are welcomed in a waiting room by the laboratory's receptionist. Waiting for sampling, they give the request for analysis and proof of payment to the receptionist who records them in the laboratory notebook.

Fourth step: sampling. The registered patient is welcomed in the phlebotomy room for sampling.

Fifth step: sample assemblage. The samples taken are recorded in a laboratory book and placed in a rack, waiting for large batch sizes.

Sixth step: Transit to laboratory i.e. transport and distribution of collected samples on appropriate benches which are either clinical biochemistry laboratory or cytohematology laboratory.

Seventh step: reception and sorting. This is the step of verification of conformity and evaluation of information and sample details. It's also the step of sample data key in and assigning laboratory IDs.

Eighth step: Sample preparation procedure. The aim of this step is to obtain an appropriate presentation of samples to the instruments of analysis. Centrifugation of blood results in obtaining serum or plasma for biochemical or hematological analyzes. Centrifugation is followed by decantation and aliquoting of the serum or plasma.

Ninth step: Analysis. Samples are analyzed manually or on automated ana- 
lyzer.

Tenth step: Result reporting. Results issuing from the laboratory analysis are completed by the technologist on the Clinical Laboratory Reporting Form.

Eleventh step: Biological validation. Only a "validated" result will be communicable. During the biological validation, the medical biologist checks the consistency of the results by taking into account various criteria: the sex and age of the patient; comparison of results with usual values; the comparison of the results between them allowing to highlight a concordance or conversely an implausibility leading the biologist to ask for verifications; Clinical and therapeutic information (signs leading to a consultation, and current treatment); Comparison of latest results with prior results.

Twelfth step: making the results available. The validated results are recorded in the laboratory results' books and entered on the computer to constitute a database. The entered results are then printed on a sheet which is signed by the doctor who validated these results. The results are thus ready to be returned to the prescriber.

As the laboratory had no laboratory information system (LIS) at the time of the study, the intra-lab TAT was quantified using a time and motion analysis approach. Standardized data collection forms were developed and distributed to trained external observers. Each observer was provided with a stopwatch and assigned to one of the five most commonly ordered tests. During round, the test assigned eyewitness recorded, for each test ordered, the time of patient reception in the laboratory, and then visually followed the test throughout the laboratory workflow process outlined (Figure 1). The external observer maintained the attention on the patient and continuously recorded the time taken to perform each of the ten laboratory workflow process steps.

\subsection{Statistical Analysis}

The collected data were entered using Microsoft Excel 2010 software. Statistical analysis was performed using SPSS version 24 (IBM, Armonk, NY). The categorical variables were tabulated as frequency and percentage. Measured TATs were checked for normality using Kolmogorov-Smirnov tests of normality. As the data were normally distributed, four measures were used to represent TAT: mean and standard deviation (SD), median and interquartile range, 90\% completion time, proportion of acceptable tests or outliers. Acceptable TAT was negotiated with attending physicians and was of $<120$ minutes for commonly ordered tests [6]. So, the evaluation of TAT consisted of comparing the overall intra-lab TAT with the suggested TAT. Statistical significance was defined at $95 \%$ confidence intervals, as $\mathrm{p}<0.05$.

\subsection{Identification of Causes of Delay}

Brainstorming was the root cause analysis tool used for identifying reasons for delay. After gathering together laboratory personnel who are familiar with TAT problems, we include one of them to act as a facilitator. This mentor wrote the 
problem on the board: "the clinical laboratory TAT is drastically longer than the suggested and recommended TAT". In order to explore all potential or real causes that result in longer TAT, the 5-Whys investigation was used as a part of the fishbone (also known as the cause-and-effect or Ishikawa) diagram as follow : a fishbone diagram was created and the six major categories of causes were written as branches from the main arrow (Figure 2). These six categories of causes, also called $6 \mathrm{M}$, are related to Machinery, Management, Manpower, Materials, Method and Milieu (Mother nature or environment). Once the $6 \mathrm{M}$ factors used to construct cause-and-effect diagrams were established on the fishbone, we used the 5-Whys technique to drill down to the root causes. The facilitator asked the first why: "why is the clinical laboratory TAT longer than suggested TAT"? Everyone in the group gave an idea in rotation (or passes his/her opportunity to give an idea) until the next round. To wrap it up, the mentor asked "why" four more times, framing each "why" in response to the answer the group had just recorded. The root cause of delay was revealed when asking "why" produces no more useful responses, and the group could go no further. Hence, the final Why leads the team to a statement (root cause) that the team can take action upon.

\subsection{Ethical Considerations}

The research proposal was submitted to the ethical review committee of the Public Health School, University of Kinshasa, and data collection was started after getting ethical clearance.

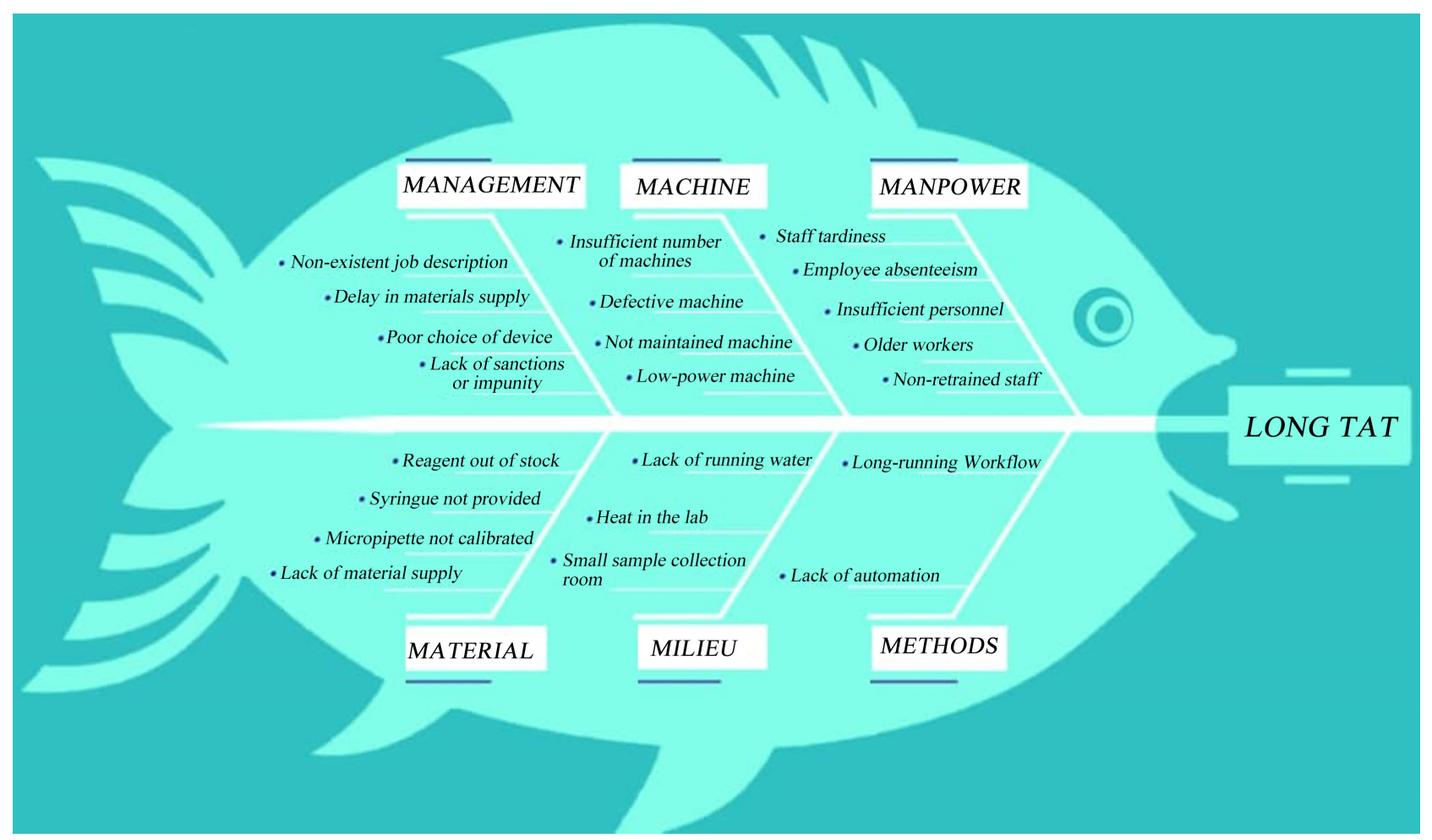

Figure 2. Root causes of long TAT. 


\section{Results}

A total of 205 samples for each type of analysis chosen (full hemogram, glucose, urea, creatinine and electrolytes), resulting in a total of 1025 samples, were included in the study.

Table 1 shows the TAT for each step of the intralaboratory workflow process and the overall TAT.

In this study, the mean total TAT was $464.4 \pm 31.05$ minutes, with a median of 393 minutes (IQR 353 - 435 minutes) and a 90\% completion time of 480 minutes for hemogram; $549.1 \pm 29.9$ minutes, with a median of 481 minutes (IQR 460 509 minutes) and a $90 \%$ completion time of 525 minutes for glucose; $483.1 \pm$ 7.32 minutes, with a median of 483 minutes (IQR 429 - 541 minutes) and a $90 \%$ completion time of 619 minutes for urea; $481 \pm 6.2$ minutes, with a median of 481 minutes (IQR 431 - 538 minutes) and a $90 \%$ completion time of 583 minutes for creatinine; $536.7 \pm 30.1$ minutes, with a median of 471 minutes (IQR 421 - 513 minutes) and a $90 \%$ completion time of 550 minutes for electrolytes. Major areas in which delays occurred were pre-analytical phase $(70 \%)$ and post-analytical phase $(25 \%)$. The processing steps that caused the biggest delay in TAT were patient reception (32\%), sample assemblage (28\%) and biological validation (15\%). Transportation of samples was the most efficient process with the mean transportation time for all the tests being around 5 minutes.

In Table 2, the TAT observed in the clinical laboratory is compared to recommended TAT for the tracked tests.

At the significance level of 0.05 , we reject the null hypothesis because p-value is less than 0.05 . The TAT observed in the laboratory is therefore higher than the suggested TAT.

Table 3 shows the distribution of the samples in different categories of TAT according to international norms and customers' suggestions.

After patient reception, only 2 (1\%) samples were reported within 60 minutes, and $3(1.5 \%)$ within 120 minutes. Outliers (or proportions of acceptable tests) = tests reported in excess of 120 minutes from the time the patient was received (ie, TATs in excess of 120 minutes.). Our study reveals an outlier rate of $98.5 \%$.

Taking into account the prolonged TAT revealed by the three previous tables, the following fishbone diagram (Figure 2) was drawn by the laboratory researchers team to try to understand the source of long TAT. The team used the six generic headings to prompt ideas. Layers of branches show thorough thinking about the causes of delay. The resulting diagram illustrates the main causes and subcauses leading to long TAT.

The total laboratory results turnaround time is influenced by many factors which has direct effects on the occurring delay. The root causes of long TAT related to Manpower (staff tardiness, employee absenteeism, insufficient personnel, older workers and non-retrained staff), Methods (long-running workflow, lack of automation), Machine (insufficient number of machines, 


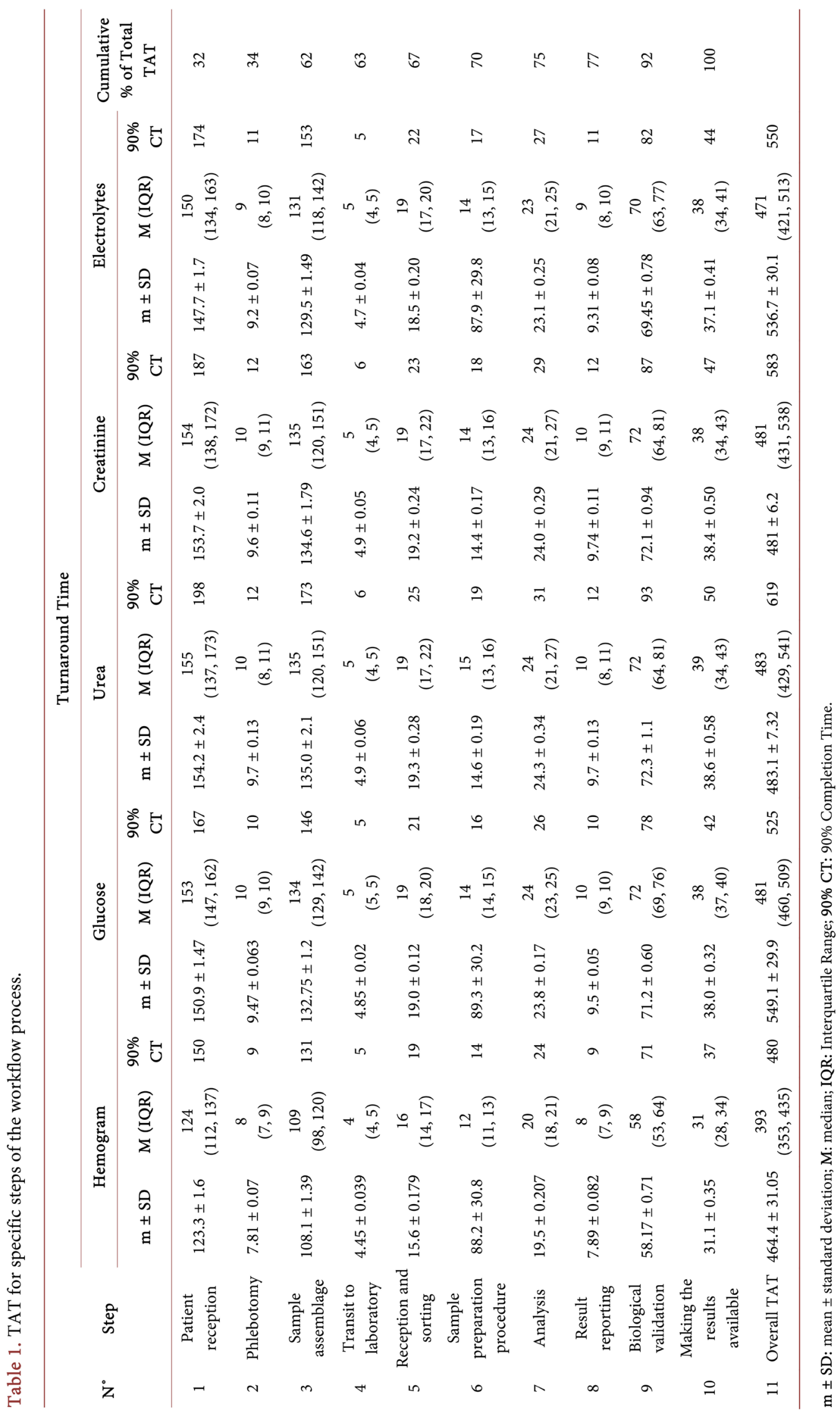


Table 2. Mean total TAT versus suggested TAT.

\begin{tabular}{cccc}
\hline Parameter & Mean \pm SD total TAT $(\mu)$ & Suggested TAT & p-value \\
\hline Hemogram & $464.4 \pm 31.05$ minutes & 120 minutes & $<0.001$ \\
Glucose & $549.1 \pm 29.9$ minutes & 120 minutes & $<0.001$ \\
Urea & $483.1 \pm 7.32$ minutes & 120 minutes & $<0.001$ \\
Creatinine & $481 \pm 6.2$ minutes & 120 minutes & $<0.001$ \\
Electrolytes & $536.7 \pm 30.1$ minutes & 120 minutes & $<0.001$ \\
\hline
\end{tabular}

Null hypothesis $\left(\mathrm{H}_{0}\right): \mu=120$ minutes Alternative hypothesis $\left(\mathrm{H}_{1}\right): \mu>120$ minutes.

Table 3. Distribution of the samples in different categories of TAT according to international norms and customers' suggestions.

\begin{tabular}{ccccccccccc}
\hline \multirow{2}{*}{ TAT } & \multicolumn{2}{c}{ Hemogram } & \multicolumn{2}{c}{ Urea } & \multicolumn{2}{c}{ Creatinine } & \multicolumn{2}{c}{ Glucose } & \multicolumn{2}{c}{ Electrolytes } \\
\cline { 2 - 11 } & $\mathbf{n}$ & $\%$ & $\mathbf{n}$ & $\%$ & $\mathbf{n}$ & $\%$ & $\mathbf{n}$ & $\%$ & $\mathbf{n}$ & $\%$ \\
\hline$\leq 60$ minutes & 2 & 0.98 & 2 & 0.98 & 2 & 0.98 & 2 & 0.98 & 2 & 0.98 \\
$61-120$ minutes & 1 & 0.49 & 1 & 0.49 & 1 & 0.49 & 1 & 0.49 & 1 & 0.49 \\
$>120$ minutes & 202 & 98.54 & 202 & 98.54 & 202 & 98.54 & 202 & 98.54 & 202 & 98.54 \\
Total & 205 & 100 & 205 & 100 & 205 & 100 & 205 & 100 & 205 & 100 \\
\hline
\end{tabular}

defective machine, not maintained machine, low-power machine), Milieu (lack of running water, heat in the laboratory, small sample collection room), Management (non-existent job description, delay in materials supply, poor choice of device, lack of sanctions or impunity), Material (reagent out of stock, syringue not provided, micropipette not calibrated, lack of material supply).

\section{Discussion}

\subsection{Discussion}

Quality can be defined as the ability of a product or service to satisfy the needs and expectations of the customer [9]. The laboratory staff generally identifies the ordering physician as the primary client. In the old days, the attending physician was only interested in the precision and accuracy of laboratory results. Nowadays, the satisfaction of the attending physician is also a function of the speed with which the laboratory performs the analyzes and returns the results to the ordering physician, in other words, the turnaround time (TAT). Lundberg defined the TAT that involves in the performance of a laboratory test as the brain to brain TAT or the "total testing cycle" [10]. The aim of this study was to measure the intra-laboratory $\mathrm{TAT}$, i.e. from patient receipt time to result electronic record time, in a tertiary hospital, and to identify reasons for delay.

The intra-lab TAT was quantified using a time and motion analysis approach. Time and motion study (also referred to as motion and time study, the terms are used interchangeably) is the analysis of the time spent in going through the different motions of a job or series of jobs. The term Time and Motion Study refers to a broad branchof knowledge dealing with the systematic determination 
of preferable work methods, with the determination of the time required for the use of human or machine to perform the work by the stipulated method, and with the development of materials required to make practical use of these data [11]. Time study consists of a wide variety of procedures for determining the amount of time required, under certain standard conditions of measurement, for tasks involving some human activity while Motion study consists of a wide variety of procedures for the description, systematic analysis, and means of improving work methods [12].

In case of laboratory TAT, we chose time and motion analysis approach to determine the amount of time required to complete each of the ten steps of the laboratory workflow process and to create a baseline that will be used in the future in order to improve the TAT. Four measures, namely mean, median, $90 \%$ completion time and outliers, were used to represent the required time. According to Steindel and Novis, these four measures are the quota to be used to adequately represent TAT [13].

However, given the long intra-lab TAT and the normal distribution of our data, the mean was used principally as it is considered to be a more objective measure in long TAT. This is founded on a recommendation by Hawkins et al. [14]. In addition, amongst the four measures of laboratory TAT, namely the mean, median, 90th percentile, and outlier rate, the mean was found to be the most reproducible of the four measures, followed by the median. The mean achieved acceptable precision with sample sizes of 100 - 500 tests [15].

Hence, in our study, the mean \pm standard deviation overall TAT was $464.4 \pm$ 31.05 minutes for hemogram, $549.1 \pm 29.9$ minutes for glucose, $483.1 \pm 7.32$ minutes for urea, $481 \pm 6.2$ minutes for creatinine and $536.7 \pm 30.1$ minutes for electrolytes (Table 1). Mahdaviazad., et al. found that the mean overall TAT varied between 1.3 - 3.1 hours [16]. Chung., et al. demonstrate the average TAT of $43.6 \pm 7.7 \mathrm{~min}$ for outpatient routine biochemistry samples [17]. A study done in Oxford determined that the TAT for hematology results was 1 hour 6 minutes (95\% CI: 29 minutes to 2 hours 13 minutes) and that for biochemistry was 1 hour 42 minutes (95\% CI: 1 hour 1 minute to 4 hours 21 minutes) [18].

In our study, the median and interquartile range (IQR) total TAT was 393 (353 - 435) minutes for hemogram, $481(460,509)$ for glucose, $483(429,541)$ for urea, $481(431,538)$ for creatinine, $471(421,513)$ for electrolytes (Table 1). As a benchmark, the 1996 Q-probes study (a nationwide survey in the United States) reported a median TAT of 130 minutes for BUN and 120 minutes for white blood cell counts [13]. In their study, Lee., et al. obtained the median total turnaround time to be $55.0(45.0$ - 69.0) $\mathrm{min}$. [19].

In the present study, the $90 \%$ Completion Time was 480 minutes for hemogram, 525 minutes for glucose, 619 minutes for urea, 583 minutes for creatinine, 550 minutes for electrolytes (Table 1). A 90\% completion time (sample registration to result reporting) of $<60$ minutes for common laboratory tests is suggested as an initial goal for acceptable TAT [14]. A study done by Steindel and Howanitz revealed that half of the 693 responding laboratories responded 
that $90 \%$ of potassium tests were ordered and reported in 69 minutes or less, whereas the TAT for $90 \%$ of haemoglobin results was 55 minutes or less [20].

The comparison of our results with those from other countries showed that the TAT in our hospital was longer than the TAT from other countries. The large differences in TATs can be explained by differences in the study settings and participants, for example, teaching vs. nonteaching hospitals, numbers of beds, and the degree of computerization in different laboratories. Evidence has it that laboratories in non-teaching hospitals and small institutions report the results more swiftly as compared to the teaching hospitals and larger institutions, respectively [7].

It was demonstrated that TAT depends on the three phases of total laboratory testing process, namely; pre-analytical, analytical and post-analytical [21]. Among these three phases, pre-analytical and post-analytical phases contribute to nearly $96 \%$ of the TAT [22]. Our results showed that pre-analytical and post-analytical phases accounted for $70 \%$ and $25 \%$ respectively, while analytical phase accounted for only $5 \%$ of the total TAT (Table 1 ). These results suggest that if the pre-analytical phase is streamlined, delayed TAT of samples could be avoided. Our findings further add to the evidence that pre-analytical and post-analytical phases of laboratory processing contribute up to $96 \%$ of total TAT.

It is generally accepted that the approuvable TAT is 1 - 2 hours [23] [24]. In our hospital, for common laboratory tests, an average of 120 minutes was suggested by users as acceptable TAT [6]. Hence, Outlier was defined as percentage of specimens exceeding suggested TAT i.e. percentage of tests reported in excess of 120 minutes from the time the patient was received by the laboratory receptionnist. Table 2 showed that the laboratory TAT is statistically greater than the suggested TAT. The TAT in our study was significantly prolonged compared to recommended TAT for the tracked tests. Table 3 revealed an outlier rate of $98.5 \%$ while Steindel [13] and Bilwani [7] reported it to be $10.4 \%$ and $2.03 \%$ respectively. Only $1.5 \%$ of our samples were reported within 120 minutes, while Chung., et al. observed $98 \%$ of the specimens being reported well within one hour [17]. In a study by Wanker, 54.65\% fell within the acceptable TAT of 60 min [25]. Bilwani., et al., in their study, found only $2.03 \%$ of the total samples being reported beyond the acceptable TAT [7]. Similarly, a study performed by Chauhan KP et al. suggested that percentage of specimens exceeding TAT was $6.4 \%$ [26].

As evident from the results, these variants of TAT in our laboratory were considerably higher than reported in many other studies. Steindel and Novis recommand that, when investigating outliers, the cause for all specimens exceeding the outlier criterion should be established [13]. Figure 2 revealed that root causes of delay related to Machinery, Management, Manpower, Materials, Method and Milieu. The major causes concerned methods and manpower (personnel). In the method category, the long-running workflow was the most offending. Workflow evaluation helps in rethinking of processes and can help 
clinical laboratories do more with less [27]. We developed a value stream map (VSM) capturing how the process is currently being done (Figure 1). The main goal of VSM is to find different types of wastes and trying to eliminate them. Waste is any step or action in a process that is not required to complete a process (called "Non Value-Adding") successfully. When waste is removed, only the steps that are required (called "Value-Adding") to deliver a satisfactory product or service to the customer remain in the process. There are eight types of waste in a work process, namely defects (work that contains errors, rework, mistakes, or lacks something necessary), overproduction (producing items for which there is no order), waiting (idle time created when material, information, people, or equipment are not ready), transport (unnecessary movements of products and materials), motion (unnecessary movements by people, eg. walking), over-processing (using more steps to produce a product than required), non-utilized talent (underutilizing employee's knowledge, skills, talents and abilities), excess inventory (holding unnecessary inventory between process steps) [28]. Table 1 shows that, among the ten workflow steps, major areas in which delays occurred were patient reception (32\%), sample assemblage (28\%) and biological validation (15\%). Waiting (waiting for sampling in the waiting room, waiting for large batch sizes after sample collection) and excess inventory (many registrations) were the two types of waste observed in these three areas. Redundant steps in the laboratory testing workflow are particularly common in low-and middle-income countries, especially in Sub-Saharan Africa settings, which commonly use paper-based laboratory service requests and results reporting [29]. The frequent use of manual methods in the analysis of samples in our laboratory significantly extends the time taken to render results. The contribution of the automatic method of analysis to the reduction of the delay in rendering results has already been demonstrated: with faster turnaround and elimination of wasteful practices, automated processes facilitate time savings [30].

Another important reason for delay in our laboratory related to manpower. Almost half of the laboratory's staff are old and can no longer work quickly. In addition, laboratory staff are often late and sometimes absent. The late arrival of the staff at the workplace, the delay in the acquisition of equipment, the delay in materials supply, the delay in the calibration of the equipment ... all these delays lead to the delay in the rendering of laboratory results. One of the most common problems business owners and managers find impacts on the effectiveness of the company is employee lateness. In a survey conducted by the Heathrow Express, employee lateness costs the UK economy around $£ 9$ billion a year. Worryingly 11 per cent say a late arrival has contributed to the loss of a potential new client. Regular lateness means that each employee loses an average of 97 minutes per month costing British employers $£ 305$ per head every year [31]. Though managers assume staff members will occasionally be late, if employees begin to be late for work regularly, then actions need to be taken to protect the company and to improve the work ethic amongst the team. When someone is chronically 
late, the natural flow of work is disrupted as other team members adjust to cover for the delay. Punctual employees that are strong and passionate are critical to productivity as well as the company's overall success. For business leaders, tardiness needs to be addressed because it affects productivity and - ultimately the reputation of an organization.

\subsection{Limitations of the Study and Suggestions for Future Research}

This study has some limitations which must be acknowledged.

First, the study is limited to samples collected during the period of 8 hours, starting from the morning, and to 5 parameters (tests) only. In addition, the research focuses only on intra-lab TAT. Thus, future research should take into account all parameters regardless of the time of sample collection. In addition, consider TAT from the request of the analysis to the rendering of results and not only intra-lab TAT.

Secondly, this preliminary study concerned only outpatients because they are the most numerous. TAT will likely be different for inpatients tests. Future research should consider inpatients tests too.

\subsection{Study Strengths}

In spite of the above limitations, this study is the first of its kind to provide data on clinical laboratory tests' TAT and to identify reasons for delay in Democratic Republic of the Congo. Through this study, we demonstrate the need to analyze TAT systematically in order to implement mechanisms to mitigate long TAT.

\section{Conclusion}

The laboratory TAT is significantly very long and does not comply with either international standards or the suggestions (requirements) of attending physicians who are the laboratory's primary clients. There are many factors that directly affect or interact with each other to affect TAT. The root causes of the delay fall into six categories and concern Machine, Management, Manpower, Material, Method and Milieu. Preventive and curative measures must be undertaken by the laboratory's manager to reduce the delay and improve the TAT.

\section{Acknowledgements}

The authors would like to acknowledge anyone who contributed towards the article, but does not meet the criteria for authorship.

\section{Conflicts of Interest}

The authors declare no conflicts of interest regarding the publication of this paper.

\section{References}

[1] Drwiega, J., Chaffin, C. and Hardy, R. (2016) Adventures in Turnaround Time. 
American Association of Clinical Chemistry, Washington DC. https://www.aacc.org/cln/articles/2016/june/adventures-in-turnaround-time

[2] Handorf, C.R. (1995) College of American Pathologists Conference XXVIII on Alternate Site Testing: Introduction. Archives of Pathology \& Laboratory Medicine, 119, 867-873.

[3] Lewandrowski, K. (2004) How the Clinical Laboratory and the Emergency Department Can Work Together to Move Patients through Quickly. Clinical Leadership \& Management Review, 18, 155-159.

[4] WHO (World Health Organization) (2015) Laboratory Quality Stepwise Implementation Tool.

https://extranet.who.int/lqsi/content/determine-turnaround-time-each-examinatio n-performed-laboratory

[5] Weinstein, S. (1995) Quality in Pathology Laboratory Practice. Journal of Quality in Clinical Practice, 15, 121-126.

[6] Alain, C.B., Rostin, M.M.M., Joël, K.N.N., Mavok Hypolite, M.M., Donatien, K.N., Koffi, T.A., et al. (2021) Evaluation of the Quality of Clinical Laboratory Services in the University Hospital of Kinshasa, Democratic Republic of the Congo. Journal of Economics and International Business Management, 9, 44-50.

https://doi.org/10.33495/jeibm_v9i1.21.145

[7] Bilwani, F., Siddiqui, I. and Vaqar, S. (2013) Determination of Delay in Turnaround Time (TAT) of Stat Tests and Its Causes: An AKUH Experience. Journal of Pakistan Medical Association, 53, 65-67.

[8] Howanitz, J.H. and Howanitz, P.J. (2001) Laboratory Results. Timeliness as a Quality Attribute and Strategy. American Journal of Clinical Pathology, 116, 311-315. https://doi.org/10.1309/H0DY-6VTW-NB36-U3L6

[9] Bergman, B.K.B. (1994) Quality: From Customer Needs to Customer Satisfaction. McGraw-Hill, New York.

[10] Lundberg, G.D. (1981) Acting on Significant Laboratory Results. JAMA, 245, 1762-1763. https://doi.org/10.1001/jama.1981.03310420052033

[11] David, L.D. and Marvin, E.M. (1994) Motion and Time Study (Paperback). Prentice Hall, Hoboken.

[12] Gilbreth, F.B. (2008) Motion Study: A Method for Increasing the Efficiency of the Workman. Kissenger Publishing, Whitefish.

[13] Steindel, S.J. and Novis, D.A. (1999) Using Outlier Events to Monitor Test Turnaround Time. A College of American Pathologist Q-Probe Study in 496 Laboratories. Archives of Pathology \& Laboratory Medicine, 123, 607-614. https://doi.org/10.5858/1999-123-0607-UOETMT

[14] Hawkins, R.C. (2007) Laboratory Turnaround Time. Clinical Biochemist Reviews, 28, 179-194.

[15] Valenstein, P.N. and Emancipator, K. (1989) Sensitivity, Specificity, and Reproducibility of Four Measures of Laboratory Turnaround Time. Am J Clin Pathol, 91, 452-457. https://doi.org/10.1093/ajcp/91.4.452

[16] Mahdaviazad, H., Javidialesaadi, F., Hosseinzadeh, M. and Masoompour, S.M. (2016) Turnaround Times for Hematology and Chemistry Tests in the Emergency Department: Experience of a Teaching Hospital in Iran. Shiraz E-Medical Journal, 17, Article No. e37101. https://doi.org/10.17795/semj37101

[17] Chung, H.J., Lee, W., Chun, S., Park, H.I. and Min, W.K. (2009) Analysis of Turnaround Time by Subdividing Three Phases for Outpatient Chemistry 
Specimens. Annals of Clinical and Laboratory Science, 39, 144-149.

[18] Gill, D., Galvin, S., Ponsford, M., Bruce, D., Reicher, J., Preston, L., et al. (2012) Laboratory Sample Turnaround Times: Do They Cause Delays in the ED? Journal of Evaluation in Clinical Practice, 18, 121-127. https://doi.org/10.1111/j.1365-2753.2010.01545.x

[19] Lee, E.J., Kim, S.C., Cho, J.S., Lee, S.C. and Park, J.O. (2011) A Point-of-Care Chemistry Test for Reduction of Turnaround and Clinical Decision Time. American Journal of Emergency Medicine, 29, 489-495. https://doi.org/10.1016/j.ajem.2009.11.020

[20] Steindel, S.J. and Howanitz, P.J. (2001) Physician Satisfaction and Emergency Department Laboratory Test Turnaround Time. Archives of Pathology \& Laboratory Medicine, 125, 863-871. https://doi.org/10.5858/2001-125-0863-PSAEDL

[21] Kilgore, M.L., Steindel, S.J. and Smith, J.A. (1998) Evaluating Stat Testing Options in an Academic Health Center: Therapeutic Turnaround Time and Staff Satisfaction. Clinical Chemistry, 44, 1597-1603.

https://doi.org/10.1093/clinchem/44.8.1597

[22] Angeletti, S., De Cesaris, M., Hart, J.G., Urbano, M., Vitali, M.A., Fragliasso, F., et al. (2015) Laboratory Automation and Intra-Laboratory Turnaround Time: Experience at the University Hospital Campus Bio-Medico of Rome. Journal of Laboratory Automation, 20, 652-658. https://doi.org/10.1177\%2F2211068214566458

[23] Steindel, S.J., Steven, J. and Bruce, A.J. (2002) Routine Outpatient Laboratory Test Turnaround Times and Practice Patterns: A College of American Pathologists Q-Probes Study. Archives of Pathology \& Laboratory Medicine, 126, 11-18. https://doi.org/10.5858/2002-126-0011-ROLTTT

[24] Goswami, B., Singh, B., Chawla, R., Gupta, V.K. and Mallika, V. (2010) Turnaround Time (TAT) as a Benchmark of Laboratory Performance. Indian Journal of Clinical Biochemistry, 25, 376-379. https://doi.org/10.1007/s12291-010-0056-4

[25] Wankar, A.D. (2014) Study of Determination of Laboratory Turnaround Time in Tertiary Care Hospital in India. International Journal of Research in Medical Sciences, 2, 1396-1401. https://doi.org/10.5455/2320-6012.ijrms20141129

[26] Chauhan, K.P., Trivedi, A.P., Patel, D., Gami, B. and Haridas, N. (2014) Monitoring and Root Cause Analysis of Clinical Biochemistry Turnaround Time at an Academic Hospital. Indian Journal of Clinical Biochemistry, 29, 505-509. https://doi.org/10.1007/s12291-013-0397-x

[27] Litvak, E. and Bisognano, M. (2011) More Patients, Less Payment: Increasing Hospital Efficiency in the Aftermath of Health Reform. Health Affairs, 30, 76-80. https://doi.org/10.1377/hlthaff.2010.1114

[28] Reddy, G.S., Lingareddy, H. and Jagadeeshwar, K. (2013) Value Stream Mapping in Manufacturing Industry. International Journal of Advanced Engineering Technology, 4, 28-30.

[29] Khan, K. (2014) Root Cause Analysis (RCA) of Prolonged Laboratory Turnaround Time in a Tertiary Care Setup. Journal of Clinical and Diagnostic Research, 8, FC05-FC08. https://doi.org/10.7860/JCDR/2014/7269.4255

[30] Tacker, D.H., Topardo, J., Mahaffey, C. and Perrotta, P.L. (2014) Workflow Analysis Comparing Manual and Automated Specimen Processing for Mass Spectrometry-Based Vitamin D Testing. Laboratory Medicine, 45, 361-367. https://doi.org/10.1309/LMZL47EN6KDODMXJ 
[31] Heathrow Express (2012) Running Late: Work Tardiness Costs UK Economy £9 Billion.

https://mediacentre.heathrow.com/pressrelease/details/81/Corporate-operational-2 $\underline{4 / 4081}$ 